Article

\title{
Acute Cystitis Symptom Score (ACSS): Clinical Validation of the Italian Version
}

\author{
Tommaso Di Vico ${ }^{1, *}$, Riccardo Morganti ${ }^{2}\left(\mathbb{D}\right.$, Tommaso Cai ${ }^{3}{ }^{(D}$, Kurt G. Naber ${ }^{4}(\mathbb{D}$, \\ Florian M.E. Wagenlehner ${ }^{5}$, Adrian Pilatz ${ }^{5}$, Jakhongir Alidjanov ${ }^{5}$, Girolamo Morelli ${ }^{6}$ and \\ Riccardo Bartoletti ${ }^{1}$ \\ 1 Department of Translational Research and New Technologies, University of Pisa, 56126 Pisa, Italy; \\ riccardo.bartoletti@hotmail.com \\ 2 SOD Clinical Trial Statistical Support, Azienda Ospedaliero Universitaria Pisana, 56126 Pisa, Italy; \\ r.morganti@ao-pisa.toscana.it \\ 3 Urology Unit, S. Chiara Regional Hospital, 38122 Trento, Italy; ktommy@libero.it \\ 4 Department of Urology, Technical University of Munich, 80333 Munich, Germany; kurt.naber@nabers.de \\ 5 Clinic of Urology, Pediatric Urology and Andrology, Justus Liebig University Giessen, 35390 Giessen, \\ Germany; Florian.Wagenlehner@chiru.med.uni-giessen.de (F.M.E.W.); \\ adrian.pilatz@chiru.med.uni-giessen.de (A.P.); jakhongir.alidjanov@med.uni-giessen.de (J.A.) \\ 6 Department of Critical Medicine, University of Pisa, 56126 Pisa, Italy; girolamomorelli@gmail.com \\ * Correspondence: tommaso.divico@yahoo.it; Tel.: +39-339-364-2655
}

Received: 30 January 2020; Accepted: 24 February 2020; Published: 2 March 2020

check for updates

\begin{abstract}
Acute Cystitis Symptom Score (ACSS) is an 18-item self-reporting questionnaire for clinical diagnosis and follow-up of acute uncomplicated cystitis (AUC) in women. The ACSS, originally developed in Uzbek and Russian languages, is now available in several languages. The purpose of the study was to validate the ACSS questionnaire in the Italian language. Linguistic validation was carried out according to Linguistic Validation Manual for Patient-Reported Outcomes Instruments guidelines. Clinical validation was carried out by enrolling one hundred Italian-speaking women. All women were asked to fill in the ACSS questionnaire during their medical visit. Fifty-four women, median age 36 (Inter Quartile Range 28-49), were diagnosed with AUC, while 46 women, median age 38 (IQR 29-45), were enrolled as the control group attending the hospital's fertility center for couples. The most frequently isolated pathogen in AUC was Escherichia coli (40;74.0\%) followed by Enterococcus faecalis $(7 ; 13.0 \%)$ and Staphylococcus saprophyticus $(3 ; 5.6 \%)$. Receiver operating characteristic (ROC) curve analysis performed at the first diagnostic visit on a typical symptoms domain cut-off score of 6 revealed a sensitivity of $92.5 \%$ and specificity of $97.8 \%$. The Italian version of the ACSS has proved to be a reliable tool with a high accuracy in diagnosis and follow-up in women with AUC. The ACSS may also be useful for clinical and epidemiological studies.
\end{abstract}

Keywords: cystitis; female; urinary tract infection; pain; questionnaire; acute cystitis symptom score; ACSS

\section{Introduction}

Acute uncomplicated cystitis (AUC) is one of the most common and widespread pathological conditions among women of all ages, with a relevant impact on social costs and quality of life [1]. Although AUC is a benign disease, recurrent episodes are associated with reduction in quality of life, everyday activities, such as social and familiar relationships, leisure time and physical activities, working ability, and psychosexual disorders [2].

The guidelines on urological infections of the European Association of Urology (EAU) do not consider urinalysis or other microbiological investigations as necessary in the case of patients with 
AUC at their first episode [3]. As a consequence, in Italy, many general practitioners' prescriptions are based just on a telephone conversation about the patients' complaints and symptoms [2,4]. Antibiotic stewardship programs aim to coordinate strategies to enhance health outcomes, decrease the use of broad-spectrum antibiotics, and slow down the increase of antimicrobial resistance $[5,6]$. For these reasons, usage of broad-spectrum antibiotics should be limited only to individual cases. On the other hand, several non-infectious diseases, such as painful bladder syndrome, urolithiasis, and overactive bladder, may also be related to the same symptoms and, in the case of recurrence after empirical antibiotic therapy, urinalysis and microbiological investigations become mandatory. However, the perception of symptoms remains of paramount importance for the diagnostic recognition of an acute cystitis episode. Different urinary symptoms have been described by patients who frequently experience recurrent episodes of cystitis. There are, however, no questionnaires available to gauge the severity of symptoms and their interference with daily activities and to monitor treatment efficacy.

The Acute Cystitis Symptom Score (ACSS) was developed under the hypothesis that the diagnosis of acute cystitis can be made with high probability based on the typical symptoms, such as frequency, urgency, and dysuria, in the absence of vaginal and/or urethral discharge [7]. In particular, the ACSS questionnaire was developed for: (a) detection and evaluation of the severity of acute cystitis symptoms, (b) assessing the impairment of everyday activities and quality of life caused by the symptoms, and (c) differentiation of AUC from other disorders that present with similar symptoms [8]. The Uzbek and Russian versions of the ACSS were originally created and tested in Uzbek- and Russian-speaking female populations of the Republic of Uzbekistan. Thereafter, the ACSS was translated into and validated in the German, British English, Hungarian, and Tajik languages (www.acss.world). The ACSS and its scoring system have demonstrated high values of reliability, validity, and discriminative abilities in all studies conducted in these countries. The Polish, Romanian, Ukrainian, Turkish, French, Portuguese, Spanish, American English, and traditional Chinese versions have been already linguistically validated and are under definite clinical validations [8-13]. Here, we aimed to validate the ACSS in the Italian language for clinical use and for epidemiological studies.

\section{Materials and Methods}

This study was designed as a prospective, observational cohort study in women with symptoms and a microbiological diagnosis of AUC, appropriately treated and monitored as recommended in the EAU guidelines [3]. A second cohort of women attending the hospital's fertility center for couples and not suspected of having a urinary tract infection (UTI) was enrolled as the control group. The two cohorts of women were considered for the clinical validation of the Italian ACSS.

\subsection{Clinical and Microbiological Considerations}

In accordance with EAU guidelines on urological infections, the diagnosis of a UTI was defined according to the following parameters: patient reported symptoms, patient interview, physical examination, bed-side dip-stick urinalysis, and urine culture [3]. In addition to the urological examinations, all women included into the study were asked to fill out the final Italian version of the ACSS questionnaire (see below). All clean-catch midstream urine samples collected at room temperature were immediately taken to the laboratory under refrigerated conditions and analyzed. All urine samples were analyzed for common uropathogenic bacteria and yeasts and aliquoted for DNA extraction and polymerase chain reaction testing for Chlamydia trachomatis, Neisseria gonorrhoeae, and urogenital Mycoplasma. For microbiological diagnosis, a colony count of $\geq 10^{5} \mathrm{CFU} / \mathrm{mL}$ was considered the cutoff for significant bacterial growth. Appropriate therapy for those women diagnosed with AUC was prescribed according to EAU guidelines [3].

\subsection{The ACSS Questionaire}

The ACSS questionnaire was developed and originally validated in the Uzbek and Russian languages as a simple and self-reported questionnaire that is helpful for the diagnosis of AUC in 
women [7,9]. The ACSS contains 18 questions (items) that are divided into 4 domains: 6 items regarding typical acute cystitis symptoms ("Typical" domain), 4 items for differential diagnosis ("Differential" domain), 3 items on quality of life ('QoL' domain), and 5 additional questions regarding other relevant circumstances, such as menstruation and pregnancy ("Additional" domain). The first 3 domains are designed and scored on a Likert-type scale in order to measure the severity of symptoms, while the items of the last domain are dichotomously designed by only requiring simple 'Yes/No' answers. The Likert-type scale included four classes of symptom severity according to the following terms: no symptoms (0), mild (1), moderate (2), severe (3) [14].

\subsection{Translation Process}

The translation and linguistic validation of the Italian version of the ACSS was performed in accordance with the Linguistic Validation Manual for Patient-Reported Outcomes (PRO) Instruments guidelines [15]. Starting from the validated UK (British) English version of the ACSS [11], two forward translations into the Italian language were produced by two professional independent translators. After a consultative meeting between the two independent translators and the local project manager, the "first consensus version" was obtained. Then, the "first consensus version" was back-translated to UK English language by an independent British English native speaker translator. This version was compared with the validated UK (British) English version of the questionnaire in order to detect, discuss, and eliminate any relevant differences. Then this "corrected provisional version" of the questionnaire was used for a cognitive assessment test carried out on 10 Italian-speaking women with different educational levels. All comments of the patients were then discussed between the translators and the local project team and the final version of the questionnaire was created (Figure 1).

\subsection{Recruitment and Validation of the Cohort Population}

All female patients (aged 18 years and older) with clinical and/or microbiological diagnosis of AUC, attending a single Italian center from November 2018 to February 2019 were enrolled as patients in our validation cohort. Patients with known diabetes mellitus or in treatment with pharmacological or herbal compounds were excluded from patient selection. In addition, women selected consecutively among those attending the hospital's fertility center for couples and not suspected for AUC were enrolled as controls. The double-blind technique was applied for the respondents' allocation into the groups. One of the urologists had access to the case histories and the results of the respondents' clinical and laboratory investigations, but he was blinded to the results of the questionnaire survey. A second urologist was blinded to all of the results of the respondents' investigations, apart from the ACSS test results. Another urologist compared the two independent diagnoses and, when their opinions coincided, true negative or true positive diagnoses were marked and the respondents were divided into two groups accordingly: the control group (Controls) and the acute cystitis group (Patients). Urinalysis with dip sticks and urine culture were performed in all subjects selected before their study enrolment. The ACSS questionnaire was administered prior (t0) and after treatment (t1) as required.

\subsection{Data Analysis and Ethical Consideration}

The categorical data were described with absolute frequency and the quantitative data with the median (interquartile range, IQR). A Kolmogorov-Smirnov test was used to asses if the quantitative data are normally distributed [16]. A Mann-Whitney two-sided test (Mann-Whitney's U test) was used to compare the quantitative variables at baseline (frequency, urgency, painful urination, incomplete emptying, suprapubic pain, and hematuria) between patients and controls [17]. Receiver operating characteristic analysis (ROC analysis), using a non-parametric test, was performed to determine the best cut-off of the typical domain total score at baseline. Sensitivity and specificity were also determined. The Wilcoxon two-sided test was used to evaluate the variation of the typical domain's variables over time [18]. Internal reliability was assessed with Spearman's rank correlation coefficient (Spearman's 
rho) [19]. All of the data obtained were analyzed using Statistical Package for the Social Sciences (IBM SPSS Statistics for Windows, Version 25.0).

\subsection{Ethical Approval}

Ethical approval was waived by the local Ethics Committee in view of the observational nature of the study and all the procedures being performed were part of the routine care. Informed consent was obtained from all individual participants included in the study.

Prima visita (modulo di diagnosi) - parte A

\section{Italiano ACSS Questionario}

\begin{tabular}{|c|c|c|c|c|c|c|}
\hline \multicolumn{7}{|c|}{ alutazione: } \\
\hline \multicolumn{7}{|c|}{ Prego indicare se hai presentato uno dei seguenti sintomi nelle ultime 24 ore e quanto sono stati intensi } \\
\hline \multicolumn{3}{|r|}{ (segnare una sola riposte per ogni sintomo) } & 0 & 1 & 2 & 3 \\
\hline \multirow{6}{*}{ 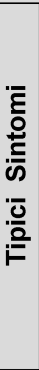 } & 1 & $\begin{array}{l}\text { Minzione frequente di piccole quantità di urina } \\
\text { (necessità di andare al bagno molto spesso) }\end{array}$ & $\begin{array}{l}\square \text { No } \\
4 \text { o meno volte } \\
\text { al giorno }\end{array}$ & $\begin{array}{l}\square \text { Sì, lieve } \\
\text { 5-6 volte al giorno }\end{array}$ & $\square_{7-8 \text { volte al giorno }}$ & $\begin{array}{l}\square \text { Sì, grave } \\
\text { 9-10 o più volte } \\
\text { al giorno }\end{array}$ \\
\hline & 2 & $\begin{array}{l}\text { Minzione urgente (desiderio intenso e incontrollabile } \\
\text { di urinare) }\end{array}$ & $\square$ No & $\square$ Sì, lieve & $\square$ Sì, moderato & $\square$ Sì, grave \\
\hline & 3 & Sensazione di dolore o bruciore durante la minzione & $\square$ No & $\square$ Sì, lieve & $\square$ Sì, moderato & $\square$ Sì, grave \\
\hline & 4 & Svuotamento vescicale incompleto dopo la minzione & $\square$ No & $\square$ Sì, lieve & $\square$ Sì, moderato & $\square$ Sì, grave \\
\hline & 5 & $\begin{array}{l}\text { Dolore o pressione fastidiosa nella parte bassa } \\
\text { dell'addome (area sovrapubica) }\end{array}$ & $\square$ No & $\square$ Sì, lieve & $\square$ Sì, moderato & $\square$ Sì, grave \\
\hline & 6 & Sangue visibile nelle tue urine & $\square$ No & $\square$ Sì, lieve & $\square$ Sì, moderato & $\square$ Sì, grave \\
\hline \multicolumn{7}{|c|}{ Somma del punteggio "tipico" $=\ldots$ punti } \\
\hline \multirow{4}{*}{ 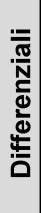 } & 7 & Dolore lombare (parte bassa della schiena) & $\square$ No & $\square$ Sì. lieve & $\square$ Sì. moderato & $\square$ Sì. arave \\
\hline & 8 & Secrezioni vaginali (soprattutto al mattino) & $\square$ No & $\square$ Sì. lieve & $\square$ Sì. moderato & $\square$ Sì. arave \\
\hline & 9 & Secrezioni uretrali (senza minzione) & $\square$ No & $\square$ Sì, lieve & $\square$ Sì, moderato & $\square$ Sì, grave \\
\hline & 10 & $\begin{array}{l}\text { Temperatura corporea elevata (brividi/febbre) } \\
\text { (si prega di indicare se misurato) }\end{array}$ & $\begin{array}{l}\square \text { No } \\
\leq 37,5^{\circ} \mathrm{C}\end{array}$ & $\begin{array}{l}\square \text { Sì, lieve } \\
37,6-37,9^{\circ} \mathrm{C}\end{array}$ & $\begin{array}{c}\square \text { Sì, moderato } \\
38,0-38,9^{\circ} \mathrm{C}\end{array}$ & $\begin{array}{c}\square \text { Sì, grave } \\
\geq 38,9^{\circ} \mathrm{C}\end{array}$ \\
\hline
\end{tabular}

Somma del punteggio "differenziale" = _ punti

11 Prego dare un punteggio complessivo su quanto i sintomi precedentemente menzionati ti hanno infastidita nelle ultime 24 ore (segnare una sola risposta)

$\square 0$ Nessun fastidio avvertito (nessun sintomo, stata bene come al solito)

$\square 1$ Sensazione di fastidio lieve (sensazione di qualcosa di peggiore rispetto al solito)

$\square 2$ Sensazione di fastidio moderato (sensazione abbastanza cattiva)

$\square 3$ Sensazione di fastidio estremo (sensazione terribile)

$\stackrel{\pi}{\stackrel{5}{5}} 12$ Prego scegliere il numero che maggiormente descrive quanto il tuo normale lavoro/attività quotidiane sono influenzate dai tuoi sintomi, sopra menzionati, nelle ultime $\mathbf{2 4}$ ore (segnare una sola risposta)

$\square 0$ Nessuna variazione (attività quotidiane svolte normalmente)

$\square 1$ Alterate lievemente (attività quotidiane svolte con qualche difficoltà)

$\square 2$ Alterate moderatamente (attività quotidiane svolte con sforzo significativo)

$\square 3$ Alterate fortemente (attività quotidiane pressoché impossibili da svolgere)

13 Indicare quanto le tue attività sociali sono state influenzate dai sintomi precedentemente menzionati nelle ultime 24 ore (segnare una sola risposta)

$\square 0$ Nessuna modifica (attività sociali svolte normalmente)

$\square 1$ Alterate lievemente (impossibilità di svolgere qualche attività sociale)

$\square 2$ Alterate moderatamente (capacità di svolgere solo qualche attività sociale)

$\square 3$ Alterate estremamente (impossibilità di svolgere nessuna attività sociale - i sintomi mi tengono "prigioniera,, in casa)

14 Indicare se oggi hai avuto una delle seguenti condizioni

Mestruazioni ? $\square$ No $\square$ Sì

Sintomi premestruali? $\quad \square$ No $\quad \square$ Sì

Sintomi della menopausa ? $\quad \square$ No $\square$ Sì

Sei incinta? $\quad \square$ No $\quad \square$ Sì

Hai il diabete mellito? $\quad \square$ No $\square$ Sì

Figure 1. Cont. 


\section{UK English ACSS Questionaire}

First visit (diagnostic form) - Part A

Time: ____ Date of evaluation:

l $I$

(dd/mm/yyyy)

\begin{tabular}{|c|c|c|c|c|c|c|}
\hline & & lease indicate whether you have had the followin & g symptoms durir & g the past 24 & ours, and how seve & ere they were: \\
\hline & & Please mark only one answer for each symptom) & 0 & 1 & 2 & 3 \\
\hline & 1 & $\begin{array}{l}\text { Frequent urination of small volumes of urine } \\
\text { (going to the toilet very often) }\end{array}$ & $\begin{array}{l}\square \text { No } \\
4 \text { or less times per day }\end{array}$ & $\square_{5-6 \text { times/day }}$ Yes, mild & $\square$ Yes, moderate & $\begin{array}{l}\square \text { Yes, severe } \\
\text { 9-10 or more times/day }\end{array}$ \\
\hline$\sum_{0}^{0}$ & 2 & $\begin{array}{l}\text { Urgent urination (a sudden and uncontrollable } \\
\text { urge to pass urine) }\end{array}$ & $\square$ No & $\square$ Yes, mild & $\square$ Yes, moderate & $\square$ Yes, severe \\
\hline के & 3 & Feeling pain or burning when passing urine & $\square$ No & $\square$ Yes, mild & $\square$ Yes, moderate & $\square$ Yes, severe \\
\hline $2^{2}$ & 4 & Incomplete bladder emptying after urination & $\square$ No & $\square$ Yes, mild & $\square$ Yes, moderate & $\square$ Yes, severe \\
\hline & 5 & $\begin{array}{l}\text { Pain or uncomfortable pressure in the lower } \\
\text { abdomen (suprapubic area) }\end{array}$ & $\square$ No & $\square$ Yes, mild & $\square$ Yes, moderate & $\square$ Yes, severe \\
\hline & 6 & Visible blood in your urine & $\square$ No & $\square$ Yes, mild & $\square$ Yes, moderate & $\square$ Yes, severe \\
\hline & & & & Sum of "1 & ypical" scores= & points \\
\hline & 7 & Loin (low back) pain* & $\square$ No & $\square$ Yes, mild & $\square$ Yes, moderate & $\square$ Yes, severe \\
\hline 耐 & 8 & Vaginal discharge (especially in the morning) & $\square$ No & $\square$ Yes, mild & $\square$ Yes, moderate & $\square$ Yes, severe \\
\hline$\frac{\bar{\omega}}{\mathrm{c}}$ & 9 & Urethral discharge (without urination) & $\square$ No & $\square$ Yes, mild & $\square$ Yes, moderate & $\square$ Yes, severe \\
\hline 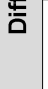 & 10 & $\begin{array}{l}\text { High body temperature (chills/fever) } \\
\text { (Please indicate if measured) }\end{array}$ & $\begin{array}{l}\square \text { No } \\
\leq 37.5{ }^{\circ} \mathrm{C}\end{array}$ & $\begin{array}{l}\square \text { Yes, mild } \\
37.6^{\circ}-37.9^{\circ} \mathrm{C}\end{array}$ & $\begin{array}{r}\square \text { Yes, moderate } \\
38.0^{\circ} \mathrm{C}-38.9^{\circ} \mathrm{C}\end{array}$ & $\begin{array}{c}\square \text { Yes, severe } \\
\geq 39.0^{\circ} \mathrm{C}\end{array}$ \\
\hline & & *often unilateral (on one side) & & Sum of "Dif & erential" scores $=$ & points \\
\hline & 11 & $\begin{array}{l}\text { Please give an overall rating of how much these sy } \\
\text { (Please mark only one answer) }\end{array}$ & mptoms, mentic & d above, bot & ed you in the past & 24 hours \\
\hline & & $\begin{array}{l}\square \text { 0 Do not feel any discomfort (No symptoms at all. } \\
\square \text { 1 Feeling little discomfort (Feeling somewhat wor } \\
\square \text { 2 Feeling moderate discomfort (Feeling quite bac } \\
\square \text { 3 Feeling extreme discomfort (Feeling terrible) }\end{array}$ & $\begin{array}{l}\text { (Felt as good as } \\
\text { rse than usual) } \\
\text { d) }\end{array}$ & & & \\
\hline 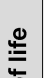 & 12 & $\begin{array}{l}\text { Please choose the number, which most closely } \\
\text { symptoms, mentioned above, in the past } 24 \text { hour }\end{array}$ & $\begin{array}{l}\text { describes your nc } \\
\text { rs (Please mark o }\end{array}$ & $\begin{array}{l}\text { mal work/ev } \\
\text { ly one answ }\end{array}$ & activities we & ected by your \\
\hline 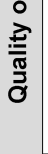 & & $\begin{array}{l}\square \text { 0 Not affected at all (Camying out usual daily activi } \\
\square \text { 1 Mildly affected (Able to carry out daily activities } \\
\square \text { 2 Moderately affected (Only able to carry our dail) } \\
\square \text { 3 Extremely affected (Almost impossible to carry }\end{array}$ & $\begin{array}{l}\text { ities) } \\
\text { with some discomfc } \\
\text { y activities with sign } \\
\text { out daily activities) }\end{array}$ & $\begin{array}{l}\text { rt) } \\
\text { ificant effort) }\end{array}$ & & \\
\hline & 13 & $\begin{array}{l}\text { Please indicate, how much your social activities } \\
\text { (Please mark only one answer) }\end{array}$ & were affected by & r sympton & ntioned above i & he past 24 hours \\
\hline & & $\begin{array}{l}\square \text { 0 Not affected at all (Able to enjoy normal social a } \\
\square 1 \text { Mildly affected (Only able to do some social act } \\
\square \text { 2 Moderately affected (Only able to do a few soci } \\
\square \text { 3 Extremely affected (Not able to do any social ac }\end{array}$ & $\begin{array}{l}\text { activities) } \\
\text { (ivities) } \\
\text { al activities) } \\
\text { tivity - symptoms }\end{array}$ & p me a "priso & her" in my home) & \\
\hline & & & & Sum of & "QoL" scores= & points \\
\hline & 14 & Please indicate whether you have the following $t$ & today & & & \\
\hline & & Menstruation (women's monthly period)? & & & $\square$ No & $\square$ Yes \\
\hline$\stackrel{0}{0}$ & & Premenstrual symptoms? & & & $\square$ No & $\square$ Yes \\
\hline$\frac{5}{0}$ & & Symptoms of the menopause? & & & $\square$ No & $\square$ Yes \\
\hline$\varangle$ & & Are you pregnant? & & & $\square$ No & $\square$ Yes \\
\hline & & Do you have diabetes mellitus (sugar diabetes) & & & $\square$ No & $\square$ Yes \\
\hline
\end{tabular}

Figure 1. ACSS Italian and UK English version [11].

\section{Results}

\subsection{Demographics}

Fifty-four female patients diagnosed with acute bacterial, uncomplicated cystitis were consecutively selected in our hospital's outpatient office, whilst 46 women attending the hospital's fertility center for couples and not suspected for UTI were considered as the control group. None of the control group patients had pyuria or nitrites at the urinary dipstick test, but several women 
had urinary symptoms due to other urological disorders, such as overactive bladder, urinary stones, interstitial cystitis, or obstructive cystocele. No bladder cancer or other neoplasms were found in both groups (Table 1).

\subsection{Microbiological Findings}

Fifty-four positive urine cultures were collected during the study in patients with AUC. Among these, the most common pathogens isolated were Escherichia coli (40) followed by Enterococcus faecalis (7), Staphylococcus saprophyticus (3), Proteus mirabilis (2), Pseudomonas aeruginosa (1), and Klebsiella pneumoniae (1). We observed that patients with Enterococcus faecalis infection had milder symptoms than patients with other microorganism infections.

Table 1. Clinical features and comparison of the factors between groups at baseline ( $\mathrm{t} 0)$.

\begin{tabular}{|c|c|c|c|c|c|c|}
\hline \multirow{2}{*}{ Age: Median (IQR) } & & \multicolumn{2}{|c|}{ Patients $(n=54)$} & \multicolumn{2}{|c|}{ Controls $(n=46)$} & \multirow[b]{3}{*}{$p$-value } \\
\hline & & \multicolumn{2}{|l|}{$38(28 ; 49)$} & \multirow[t]{2}{*}{$36.5(25 ; 44)$} & \multirow[b]{2}{*}{ Median (IQR) } & \\
\hline & Likert Scale & & Median (IQR) & & & \\
\hline & 0 (no) & 0 & & 33 & & \multirow{4}{*}{$<0.0001$} \\
\hline Frequency $(\mathrm{n})$ & 1 (mild) & 17 & $1(1 \cdot 2)$ & 11 & $0(0 ; 1)$ & \\
\hline & 2 (moderate) & 22 & $1(1 ; 2)$ & 2 & $0(0 ; 1)$ & \\
\hline & 3 (severe) & 15 & & 0 & & \\
\hline \multirow{4}{*}{ Urgency (n) } & 0 (no) & 0 & \multirow{4}{*}{$2(1 ; 2)$} & 30 & \multirow{4}{*}{$0(0 ; 1)$} & \multirow{4}{*}{$<0.0001$} \\
\hline & 1 (mild) & 16 & & 10 & & \\
\hline & 2 (moderate) & 28 & & 6 & & \\
\hline & 3 (severe) & 10 & & 0 & & \\
\hline \multirow{4}{*}{ Painful Urination (n) } & 0 (no) & 7 & \multirow{4}{*}{$1(1 ; 2)$} & 33 & \multirow{4}{*}{$0(0 ; 1)$} & \multirow{4}{*}{$<0.0001$} \\
\hline & 1 (mild) & 24 & & 12 & & \\
\hline & 2 (moderate) & 14 & & 1 & & \\
\hline & 3 (severe) & 9 & & 0 & & \\
\hline \multirow{4}{*}{ Incomplete Emptying (n) } & 0 (no) & 6 & \multirow{4}{*}{$1(1 ; 1)$} & 35 & & \\
\hline & 1 (mild) & 29 & & 10 & $0(0 \cdot 0)$ & $<00001$ \\
\hline & 2 (moderate) & 13 & & 1 & $0(0,0)$ & $<0.0001$ \\
\hline & 3 (severe) & 6 & & 0 & & \\
\hline & 0 (no) & 6 & & 38 & & \\
\hline Soprapubic Pain (n) & 1 (mild) & 26 & $1(1 \cdot 2)$ & 7 & $0(0 \cdot 0)$ & $<00001$ \\
\hline Soprapuoric I aill (II) & 2 (moderate) & 16 & $1(1 ; 2)$ & 1 & $0(0 ; 0)$ & $<0.0001$ \\
\hline & 3 (severe) & 6 & & 0 & & \\
\hline & 0 (no) & 6 & & 43 & & \\
\hline Hematuria (n) & 1 (mild) & 32 & $1(1: 1)$ & 2 & $0(0 ; 0)$ & $<0.0001$ \\
\hline Hematuria (n) & 2 (moderate) & 15 & $1(1 ; 1)$ & 1 & $0(0 ; 0)$ & $<0.0001$ \\
\hline & 3 (severe) & 16 & & 0 & & \\
\hline & 0 (no) & 25 & & 44 & & \\
\hline Flank Pain (n) & 1 (mild) & 13 & $1(0 \cdot 2)$ & 2 & & \\
\hline Flank Pain (n) & 2 (moderate) & 16 & $1(0 ; 2)$ & 0 & $0(0 ; 0)$ & $<0.0001$ \\
\hline & 3 (severe) & 0 & & 0 & & \\
\hline & 0 (no) & 19 & & 46 & & \\
\hline Vaginal Discharge (n) & 1 (mild) & 16 & $1(0 \cdot 2)$ & 0 & $0(0 \cdot 0)$ & 00003 \\
\hline vagillar Disctiarge (II) & 2 (moderate) & 18 & $1(0,2)$ & 0 & $0(0,0)$ & 0.0003 \\
\hline & 3 (severe) & 0 & & 0 & & \\
\hline & 0 (no) & 19 & & 38 & & \\
\hline Urethral Discharge (n) & 1 (mild) & 24 & & 7 & & \\
\hline Uretnral Discrnarge (n) & 2 (moderate) & 9 & $1(0 ; 1)$ & 1 & $0(0 ; 0)$ & $<0.0001$ \\
\hline & 3 (severe) & 2 & & 0 & & \\
\hline & 0 (no) & 20 & & 46 & & \\
\hline Feeling Feverish (n) & 1 (mild) & 23 & $1(0 ; 1)$ & 0 & $0(0 \cdot 0)$ & \\
\hline & 2 (moderate) & 11 & $1(0 ; 1)$ & 0 & $0(0 ; 0)$ & $<0.0001$ \\
\hline & 3 (severe) & 0 & & 0 & & \\
\hline & 0 (no) & 1 & & 40 & & \\
\hline General Discomfort (n) & 1 (mild) & 14 & $2(1 \cdot 3)$ & 6 & $0(0 \cdot 0)$ & $<00001$ \\
\hline 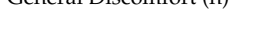 & 2 (moderate) & 21 & $2(1,0)$ & 0 & $0(0,0)$ & $<0.0001$ \\
\hline & 3 (severe) & 18 & & 0 & & \\
\hline & 0 (no) & 6 & & 42 & & \\
\hline Impact on Everyday & 1 (mild) & 16 & $2(1 ; 2)$ & 4 & $0(0 ; 0)$ & $<0.0001$ \\
\hline Activity (n) & 2 (moderate) & 23 & $2(1 ; 2)$ & 0 & $0(0 ; 0)$ & $<0.0001$ \\
\hline & 3 (severe) & 9 & & 0 & & \\
\hline & 0 (no) & 1 & & 40 & & \\
\hline Impact on Social Life (n) & 1 (mild) & 15 & $2(1: 3)$ & 5 & $0(0 \cdot 0)$ & $<00001$ \\
\hline 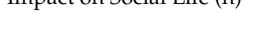 & 2 (moderate) & 18 & $2(1,0)$ & 1 & $0(0,0)$ & $<0.0001$ \\
\hline & 3 (severe) & 20 & & 0 & & \\
\hline
\end{tabular}

The median (IQR) age of the Controls and Patients was 36 (28-49) and 38 (29-45) years, respectively. 


\subsection{Validity and Reliability}

Spearman's rho, calculated between the items of the typical domain was $0.276(p=0.044)$ and, at the same time, Pearson' $\mathrm{r}$ was $0.3(p=0.027)$. ROC analysis was performed on the "Typical Domain" score in order to evaluate the questionnaire's discriminative ability. For the prediction of acute cystitis, a cut-off score of 5.5 of the typical domain showed the best balanced sensitivity (94\%) and specificity (98\%), respectively (Figures 2 and 3). Since no individual patient can have a total score of 6, we also calculated these parameters for total scores of 5 (sensitivity 95.3\%, specificity 95.7\%) and 6 (sensitivity $92.5 \%$, specificity $97.8 \%$ ).

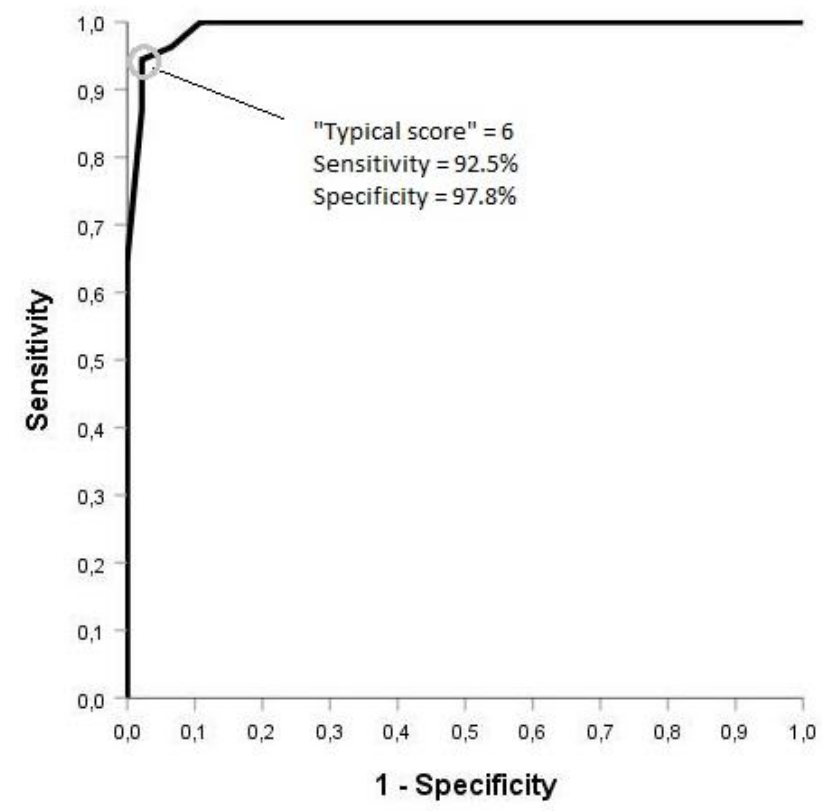

Figure 2. ROC analysis on the "Typical Domain" score at baseline.

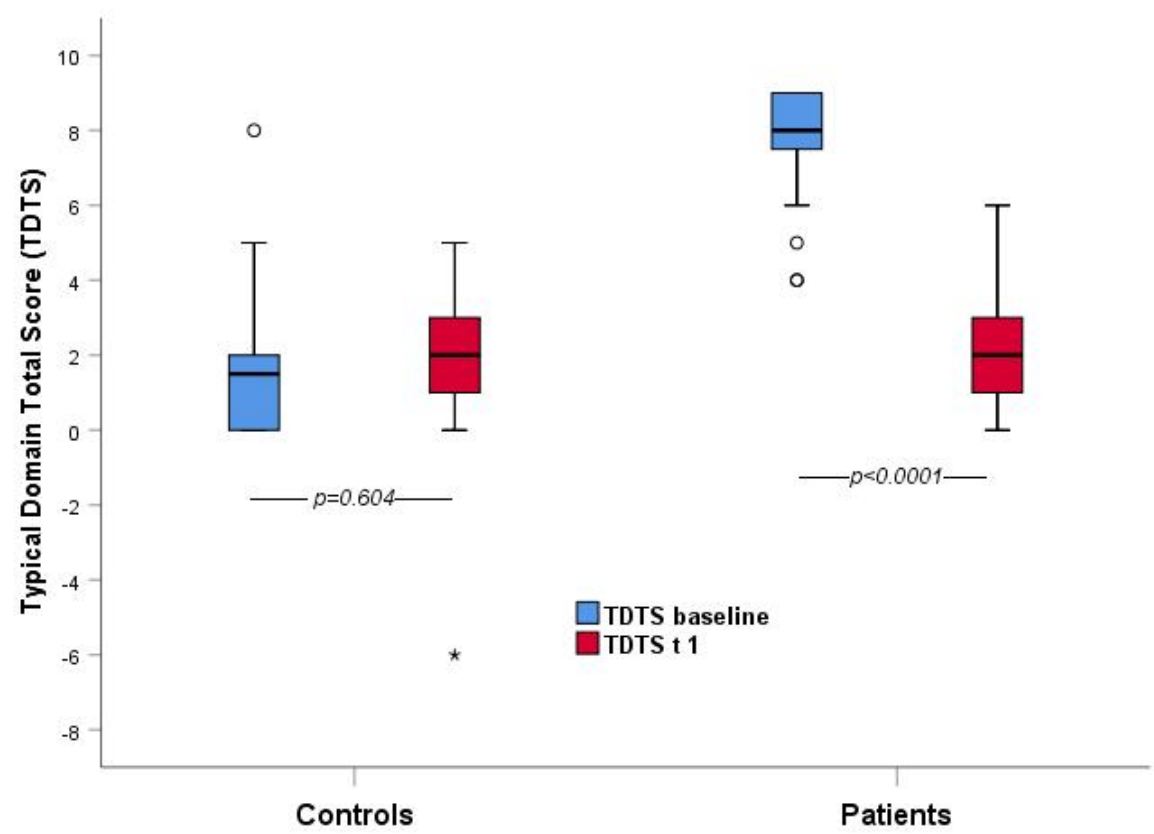

Figure 3. Comparison of "Typical Domain" total score between patients and controls at the baseline and $\mathrm{T} 1$ (the average interval between the two visits was 10 days). 


\subsection{Comparative Analysis}

Comparative analysis showed statistically significant differences between the patient's and control's baseline scores in each item belonging to the typical, differential, and quality of life domains at $p<0.05$ (except for "feeling feverish" $p=0.35$ ). Statistical significance for the domain mentioned above was also found for the difference between the first and second visit in female patients diagnosed with AUC (except for the item "feeling feverish" $p=0.356$ ).

\section{Discussion}

\subsection{Main Findings}

This Italian version of the ACSS has proven to be useful to diagnose AUC in women due to the statistically significant correlations between patients with AUC and the controls without AUC in the typical domain scores and the results of the urinalyses. Although urinary symptoms may be typical for acute cystitis, they are, however, not specific because they are also found in patients with other urological disorders. [20,21]. Therefore, not only their presence but also their severity need to be considered. The distinctiveness of the ACSS questionnaire is the assessment of the severity of symptoms, their effect on quality of life, and the differentiation of cystitis from other urogenital disorders.

Some of the women included in the control group reported urinary symptoms, such as mild or moderate frequency, urgency, and painful urination, but no significant bacteriuria, as defined above, was found in the urine culture test. The ACSS questionnaire was also able to differentiate subjects with AUC from patients with painful bladder syndrome/interstitial cystitis, vulvodynia, overactive bladder, or other non-bacterial diseases in all cases, thus demonstrating its efficient clinical applicability. In a retrospective study on 442 patients Sun and Harlow [21] demonstrated that $44.8 \%$ of patients with vulvodynia had a history of UTI.

\subsection{Results in the Context of Previous Studies and Clinical Applicability}

The Italian version of the ACSS showed a sensitivity of $94 \%$ and $92.5 \%$ and a specificity of $98 \%$ and $97.8 \%$ at a total score for the "Typical" domain of 5.5 and 6, respectively, in line with the validation in other languages. The Hungarian ACSS version, for example, showed a sensitivity of $90 \%$ and a specificity of $97 \%$ at a total score of 6 for the "Typical" domain [12]. The results are also quite similar to the original Uzbek and Russian versions of the ACSS [8,9].

In the era of antibiotic crises and bacterial antibiotic resistance, an easy and reliable tool for improving diagnostic accuracy and antibiotic prescription is urgently required. All international guidelines on urological infections recommend the use of empirical treatment on the basis of local epidemiology of bacterial resistance and antibiotic stewardship programs. The first and the most important step, however, is the correct diagnosis of AUC. EAU guidelines suggest performing a correct assessment of all patient's symptom and risk factors, but no validated and self-administered questionnaire for clinical diagnosis was available so far in the Italian language. Such a questionnaire could also be used for self-diagnosis and self-assessment of patient-reported outcomes to compare the efficacy of different treatment modalities, such as antibiotic and non-antibiotic therapies. In 2016, Alidjanov et al. developed and validated the first version of the Acute Cystitis Symptom Score (ACSS) in Uzbekistan in the Uzbek and Russian languages [7,8]. This questionnaire has already been translated and clinically validated in Russian, British English, German, Hungarian, and Tajik languages, with hundreds of women enrolled [14]. The linguistically- and clinically-validated Italian version of the ACSS is now also available for the diagnosis and management of women affected by AUC and could also be used in everyday clinical practice for improving the adherence to the principles of antibiotic stewardship as well as for clinical and epidemiological trials. 


\subsection{Study Limitations}

This study showed few limitations to take into account. One is the single-center design of the study. Even if performed in a single center, the study is representative of the Italian women population due to the high variability in subjects born or permanently resident in other areas of the country and having moved by chance to this touristic city.

Another limitation may be seen in the subjective response when using a self-administered questionnaire due to individual interpretation of the questions asked. To minimize this possible limitation, the Italian version of the ACSS was not only forward and backward translated from English to Italian by two independent professionals, according to international rules (see certifications in the supplementary materials), but was finally established after an intensive cognitive assessment procedure interviewing 10 women with Italian as their mother language and with different educational levels.

A third limitation may be seen in the difficult clinical differentiation from other urological disorders, such as overactive bladder, urinary stones, interstitial cystitis, or obstructive cystocele, that report similar symptoms, which only could be differentiated from AUC by a positive urine culture [21,22]. However, the main clinical differences, besides a positive urine culture, are the acute onset and severity of typical symptoms in the case of AUC compared to the other urological disorders.

\section{Conclusions}

The ACSS questionnaire has proven to be a simple, easy, and affordable diagnostic tool that can be used in general medical practice and research, such as in well-designed clinical and epidemiological studies [23], for the diagnosis of AUC and as a patient-reported outcome measure.

Supplementary Materials: Supplementary Materials are available online at http://www.mdpi.com/2079-6382/9/3/ 104/s1.

Author Contributions: J.A.: Protocol development; R.B.: Data collection or management, manuscript writing; T.C.: Manuscript editing; T.D.V.: Data collection or management, data analysis, manuscript writing; R.M.: Data analysis; G.M.: Data collection or management; K.G.N.: Project development, manuscript editing; A.P.: Manuscript editing; F.M.E.W.: Manuscript editing. All authors have read and agreed to the published version of the manuscript.

Funding: This research received no external funding.

Conflicts of Interest: T.D.V., R.M., T.C., G.M., and R.B. declare no conflict of interest. K.G.N., F.M.E.W., A.P., and J.A. are authors and copyright holders of the ACSS questionnaire.

Copyright and Translations of the ACSS in Other Languages: The ACSS is copyrighted by the Certificate of Deposit of Intellectual Property in Fundamental Library of Academy of Sciences of the Republic of Uzbekistan, Tashkent (Registration number 2463; 26 August 2015) and the Certificate of the International Online Copyright Office, European Depository, Berlin, Germany (Nr. EU-01-000764; 21 October 2015). The rightsholders are Jakhongir Fatikhovich Alidjanov (Uzbekistan), Ozoda Takhirovna Alidjanova (Uzbekistan), Adrian Martin Erich Pilatz (Germany), Kurt Guenther Naber (Germany), Florian Martin Erich Wagenlehner (Germany).

\section{References}

1. Foxman, B.; Brown, P. Epidemiology of urinary tract infections: Transmission and risk factors, incidence, and costs. Infect. Dis. Clin. North Am. 2003, 17, 227-241. [CrossRef]

2. Colgan, R.; Keating, K.; Dougouih, M. Survey of Symptom Burden in Women with Uncomplicated Urinary Tract Infections M. Clin. Drug Investig. 2004, 24, 55. [CrossRef]

3. Bonkat, G.; Bartoletti, R.; Bruyère, F.; Cai, T.; Geerlings, S.E.; Köves, B.; Schubert, S.; Wagenlehner, F.; Wagenlehner Guidelines Associates; Mezei, T.; et al. Guidelines EA. In Proceedings of the EAU Annual Congress Barcelona 2019, Barcelona, Spain, 15-19 March 2019; ISBN 978-94-92671-04-2.

4. Galatti, L.; Sessa, A.; Mazzaglia, G.; Pecchioli, S.; Rossi, A.; Cricelli, C.; Caputi, A.P. Antibiotic prescribing for acute and recurrent cystitis in primary care: A 4 year descriptive study. J. Antimicrob. Chemother. 2006, 57, 551-556. [CrossRef]

5. Allerberger, F.; Gareis, R.; Jindrák, V.; Struelens, M.J. Antibiotic stewardship implementation in the EU: The way forward. Expert Rev. Anti-Infect. Ther. 2009, 7, 1175-1183. [CrossRef] 
6. Lesprit, P.; Brun-Buisson, C. Current Opinion in Infectious Diseases. Hosp. Antibiot. Steward. 2008, 21, 344-349. [CrossRef]

7. Alidjanov, J.F.; Naber, K.G.; Abdufattaev, U.A.; Pilatz, A.; Wagenlehner, F.M. Reevaluation of the Acute Cystitis Symptom Score, a Self-Reporting Questionnaire. Part I. Development, Diagnosis and Differential Diagnosis. Antibiotics 2018, 7, 6. [CrossRef]

8. Alidjanov, J.F.; Abdufattaev, U.A.; Makhsudov, S.A.; Pilatz, A.; Akilov, F.A.; Naber, K.G.; Wagenlehner, F.M. New Self-Reporting Questionnaire to Assess Urinary Tract Infections and Differential Diagnosis: Acute Cystitis Symptom Score. Urol. Int. 2014, 92, 230-236. [CrossRef]

9. Alidjanov, J.F.; Abdufattaev, U.A.; Makhmudov, D.; Mirkhamidov, D.; Khadzhikhanov, F.A.; Azgamov, A.V.; Akilov, F.A. Development and clinical testing of the Russian version of the Acute Cystitis Symptom Score-ACSS. Urologiia 2014, 6, 14-22.

10. Alidjanov, J.F.; Pilatz, A.; Abdufattaev, U.A.; Wiltink, J.; Weidner, W.; Naber, K.G.; Wagenlehner, F. German validation of the acute cystitis symptom score. Der Urol. 2015, 54, 1269-1276. [CrossRef]

11. Alidjanov, J.F.; Lima, H.A.; Pilatz, A.; Pickard, R.; Naber, K.G.; Safaev, Y.U.; Wagenlehner, F.M. Preliminary Clinical Validation of the UK English Version of the Acute Cystitis Symptom Score in UK English-speaking female population of the Newcastle, Great Britain. JOJ Uro Nephron 2017, 1, 555561.

12. Magyar, A.; Alidjanov, J.; Pilatz, A.; Nagy, K.; Arthanareeswaran VK, A.; Póth, S.; Köves, B. The role of the Acute Cystitis Symptom Score questionnaire for research and antimicrobial stewardship. Validation of the Hungarian version. Cent. Eur. J Urol. 2018, 71, 134-141. [CrossRef]

13. ACSS Home. Available online: http://www.ACSS.world (accessed on 30 January 2020).

14. Likert, R. A Technique for the Measurement of Attitudes. Arch. Psychol. 1932, 140, 1-55.

15. Koller, M.; West, K. Linguistic Validation Manual for Patient-Reported Outcomes (PRO) Instruments. Qual. Life Res. 2004, 14, 1791. [CrossRef]

16. Stephens, M.A. Test of fit for the logistic distribution based on the empirical distribution function. Biometrika 1979, 66, 591-595. [CrossRef]

17. Mann, H.B. On a Test of Whether One of Two Random Variables is Stochastically Larger than the other. Ann. Math. Statist. 1947, 18, 50-60. [CrossRef]

18. Wilcoxon, F. Individual comparisons by ranking methods. Biom. Bull. 1937, 1, 80-83. [CrossRef]

19. Spearman, C. The proof and measurement of association between two things. Am. J. Psychol. 1904, 15, 72-101. [CrossRef]

20. Castro-Diaz, D.; Cardozo, L.; Chapple, C.R.; Espuña, M.; Kelleher, C.; Kirby, M.; Tubaro, A. Urgency and pain in patients with overactive bladder and bladder pain syndrome. What are the differences? Int. J. Clin. Pract. 2014, 3, 356-362. [CrossRef]

21. Sun, Y.; Harlow, B.L. The association of vulvar pain and urological urgency and frequency: Findings from a community based case-control study. Int. Urogyn. J. 2019, 30, 1871-1878. [CrossRef]

22. Safdar, N.; Abbo, L.M.; Knoblock, M.; Seo, S.K. Research methods in healthcare epidemiology: Survey and qualitative research. Infect. Control Hosp. Epidemiol. 2016, 37, 1272-1277. [CrossRef]

23. Alidjanov, J.F.; Naber, K.G.; Pilatz, A.; Radzhabov, A.; Zamuddinov, M.; Magyar, A.; Tenke, P.; Wagenlehner, F.M. Evaluation of the draft guidelines proposed by EMA and FDA for the clinical diagnosis of acute uncomplicated cystitis in women. World J. Urol. 2020, 38, 63-72. [CrossRef] [PubMed]

(C) 2020 by the authors. Licensee MDPI, Basel, Switzerland. This article is an open access article distributed under the terms and conditions of the Creative Commons Attribution (CC BY) license (http://creativecommons.org/licenses/by/4.0/). 